\title{
GENERALIZED SPECTRUM AND COMMUTING COMPACT PERTURBATIONS
}

\author{
by VLADIMIR RAKOČEVIĆ
}

(Received 23rd October 1990)

\begin{abstract}
Let $X$ be an infinite-dimensional complex Banach space and denote the set of bounded (compact) linear operators on $X$ by $B(X)(K(X))$. Let $N(A)$ and $R(A)$ denote, respectively, the null space and the range space of an element $A$ of $B(X)$. Set $R\left(A^{\infty}\right)=\bigcap_{n} R\left(A^{n}\right)$ and $k(A)=\operatorname{dim} N(A) /\left(N(A) \cap R\left(A^{\infty}\right)\right)$. Let $\quad \sigma_{g}(A)=$ $\mathbb{C} \backslash\{\lambda \in \mathbb{C}: R(A-\lambda)$ is closed and $k(A-\lambda)=0\}$ denote the generalized (regular) spectrum of $A$. In this paper we study the subset $\sigma_{g b}(A)$ of $\sigma_{g}(A)$ defined by $\sigma_{\theta b}(A)=\mathbb{C} \backslash\{\lambda \in \mathbb{C}: R(A-\lambda)$ is closed and $k(A-\lambda)<\infty\}$. Among other things, we prove that if $f$ is a function analytic in a neighborhood of $\sigma(A)$, then $\sigma_{g b}(f(A))=f\left(\sigma_{g b}(A)\right)$.
\end{abstract}

1991 Mathematics subject classification scheme: 47 A53, 47 A55.

\section{Introduction and preliminaries}

Let $X$ be an infinite-dimensional complex Banach space and denote, respectively, the set of bounded, compact and finite dimensional operators on $X$ by $B(X), K(X)$ and $F(X)$. For $A$ in $B(X)$ throughout this paper $N(A)$ and $R(A)$ will denote, respectively, the null space and the range space of $A$. Set $N\left(A^{\infty}\right)=\bigcup_{n} N\left(A^{n}\right), R\left(A^{\infty}\right)=\bigcap_{n} R\left(A^{n}\right)$, $\alpha(A)=\operatorname{dim} N(A), \beta(A)=\operatorname{dim} X / R(A)$ and $k(A)=\operatorname{dim} N(A) /\left(N(A) \cap R\left(A^{\infty}\right)\right)$. Recall that an operator $A \in B(X)$ is semi-Fredholm if $R(A)$ is closed and at least one if $\alpha(A)$ and $\beta(A)$ is finite. For such an operator we define an index $i(A)$ by $i(A)=\alpha(A)-\beta(A)$. Let $\Phi_{+}(X)$ $\left(\Phi_{-}(X)\right)$ denote the set of semi-Fredholm operators with $\alpha(A)<\infty(\beta(A)<\infty)$ and $\sigma_{e k}(A)$ Kato's essential spectrum of $A$, i.e., $\sigma_{e k}(A)=\left\{\lambda \in \mathbb{C}: A-\lambda \notin \Phi_{+}(X) \cup \Phi_{-}(X)\right\}$. Furthermore, let $\sigma(A), \quad \sigma_{a}(A)$ and $\sigma_{a b}(A)=\bigcap\left\{\sigma_{a}(A+K): K \in K(X)\right.$ and $\left.A K=K A\right\}$ denote, respectively, the spectrum, the approximate point spectrum and Browder's essential approximate point spectrum of $A([17])$.

Set $V(X)=\{A \in B(X): R(A)$ is closed and $k(A)<\infty\}$ and $V_{n}(X)=\{A \in V(X): k(A)=n\}$, $n=0,1,2, \ldots$. Let us remark that $k(A)=n<\infty$ precisely when $A$ has Kaashoek's property $P(I, n)([6$, pp. $452-453])$, or when $A$ has almost uniform descent ([5, Definition 1.3]). In particular $k(A)=0$ if and only if Kato's number $v(A: I)=\infty([9$, pp. 289-290]), i.e., if and only if $N\left(A^{\infty}\right) \subset R\left(A^{\infty}\right)$. Recall that $\Phi_{+}(X) \cup \Phi_{-}(X) \subset V(X)([5$, Theorem 3.7], [10, p. 197, Example 4]). Let $\sigma_{\theta}(A)=\left\{\lambda \in \mathbb{C}: A-\lambda \notin V_{0}(X)\right\}$ denote the generalized (regular) spectrum of $A([1,10,13]) . \sigma_{g}(A)$ is a non-empty compact subset of the set of complex numbers $\mathbb{C}$.

In this paper we study the subset $\sigma_{g b}(A)$ of $\sigma_{g}(A)$ defined by 


$$
\sigma_{g b}(A)=\{\lambda \in \mathbb{C}: A-\lambda \notin V(X)\} .
$$

The relation between $\sigma_{g}(A)$ and $\sigma_{g b}(A)$ that is exhibited in this paper resembles the relation between the $\sigma_{a}(A)$ and the $\sigma_{a b}(A)$, and it is reasonable to call $\sigma_{g b}(A)$ Browder's essential generalized spectrum of $A$.

First in Section 2 we prove a Kato-type decomposition theorem for operators in $V(X)$ which is related to Kato's theorem for semi-Fredholm operators ([9, Theorem 4], [19, Proposition 2.5]).

In Section 3 we characterize $\sigma_{g b}(A)$ (Theorem 3.1) and derive several corollaries.

In Section 4 we prove that if $f$ is a function analytic in a neighborhood of $\sigma(A)$, then $\sigma_{g b}(f(A))=f\left(\sigma_{g b}(A)\right)$.

Finally, in Section 5 we investigate connected components of the set $\mathbb{C} \backslash \sigma_{g b}(A)$.

\section{A Kato-type decomposition theorem}

Theorem 2.1. Let $A \in B(X)$ be an operator with closed range. Then, $k(A)$ is finite if and only if the space $X$ decomposes into the direct sum of two closed subspaces $X_{0}$ and $X_{1}$ which are A-invariant and have the following properties:

(i) if $A_{0}$ is the restriction of $A$ to $X_{0}$ considered as an operator from $X_{0}$ to itself, then $N\left(A_{0}\right) \subset R\left(A_{0}^{\infty}\right)$,

(ii) the space $X_{1}$ is finite-dimensional and $A$ is nilpotent on it.

Proof. Suppose that the operator $A$ satisfies conditions (i) and (ii). If $A_{1}$ is the restriction of $A$ to $X_{1}$ considered as an operator from $X_{1}$ to itself, then there is an integer $n$ such that $A_{1}^{n}=0$. Also, we have $N(A)=N\left(A_{0}\right) \oplus N\left(A_{1}\right)$ and $R\left(A^{\infty}\right)=R\left(A_{0}^{\infty}\right) \subset X_{0}$. By $[5, \quad$ Lemma $2.1(\mathrm{a})] \quad N(A) \cap R\left(A^{\infty}\right)=\left[N\left(A_{0}\right) \oplus N\left(A_{1}\right)\right] \cap R\left(A^{\infty}\right)=N\left(A_{0}\right) \oplus$ $\left[N\left(A_{1}\right) \cap R\left(A^{\infty}\right)\right]=N\left(A_{0}\right)$. Hence $\operatorname{dim}\left[N(A) /\left(N(A) \cap R\left(A^{\infty}\right)\right)\right]=\operatorname{dim} N\left(A_{1}\right)$ is finite, and $k(A)<\infty$.

Conversely, suppose that $k(A)=p$ is finite. Then, by [5, Theorem 3.8] $R\left(A^{n}\right)$ is closed for each positive integer $n$, and there are $p$ vectors $x_{k, 1}, k=1, \ldots, p$, in $N(A)$ which are linearly independent modulo the subspace $N(A) \cap R\left(A^{\infty}\right)$. Now, as in [8] and [12] there are $p$ finite chains associated with $x_{k, 1}, k=1, \ldots, p$, i.e., there are vectors

$$
x_{k, 1}, \ldots, x_{k, r_{k}}, \quad(k=1, \ldots, p)
$$

such that $A x_{k, m}=x_{k, m-1}\left(m=2, \ldots, r_{k} ; k=1, \ldots, p\right)$ and $A x_{k, 1}=0(k=1, \ldots, p)$. By $[8]$ the adjoint operator $A^{*}$ of $A$ has exactly $p$ elements in $N\left(A^{*}\right)$, say $x_{k, 1}^{*}, k=1, \ldots, p$, with finite chains. Moreover, the chain associated with $x_{k, 1}^{*}$ has the same number of elements as the corresponding chain associated with $x_{k, 1}$ for each $k=1, \ldots, p$. Thus, there are elements

$$
x_{k, 1}^{*}, \ldots, x_{k, r_{k}}^{*}, \quad(k=1, \ldots, p)
$$

in the dual space $X^{*}$ of $X$ such that $A^{*} x_{k, m}^{*}=x_{k, m-1}^{*}\left(m=2, \ldots, r_{k} ; k=1, \ldots, p\right)$ and 
$A^{*} x_{k, 1}^{*}=0(k=1, \ldots, p)$. Again, by [8] we can choose functionals in (2) such that the vectors in (2) and (1) are biorthogonal; i.e., $x_{k, r_{k}-j+1}^{*}\left(x_{m, i}\right)=1$, if $k=m$ and $j=i$, $x_{k, r_{k}-j+1}^{*}\left(x_{m, i}\right)=0$ in the other cases. Let $X_{1}$ be the subspace in $X$ spanned by vectors in (1) and $X_{0}=\bigcap\left\{N\left(x_{k, m}^{*}\right): k=1, \ldots, p ; m=1, \ldots, r_{k}\right\} . X_{0}$ and $X_{1}$ are closed subspaces in $X$, and by [14, pp. 150-151] we have $X=X_{0} \oplus X_{1}$. It is easy to see that $X_{1}$ is a finite dimensional space which is $A$-invariant and that $A$ is nilpotent on it. Further, by [12, Remark] the subspace $X_{0}$ is invariant. Next, $R\left(A^{n}\right)$ is closed for each positive integer $n$ [5, Theorem 3.8], by the proof of [12, Theorem 5] we have $N\left(A_{0}\right) \subset R\left(A_{0}^{\infty}\right)$. This completes the proof.

Remark 2.2. Since $R(A)$ is closed subspace in $X$, and $R(A)=R\left(A_{0}\right) \oplus R\left(A_{1}\right)$ by [9, Lemma 3.32] $R\left(A_{0}\right)$ is a closed subspace in $X$.

Remark 2.3. By [19, Lemma 1.3 and Corollary 1.4] we have $k\left(A_{0}\right)=0$. Thus, by Remark 2.2, $A_{0} \in V_{0}\left(X_{0}\right)$.

\section{Characterization of $\sigma_{g b}(\mathrm{~A})$}

Theorem 3.1. Let $A \in B(X)$. Then

$$
\sigma_{g b}(A)=\bigcap\left\{\sigma_{g}(A+K): K \in K(X) \text { and } A K=K A\right\} .
$$

Proof. If $\lambda \notin \bigcap\left\{\sigma_{g}(A+K): K \in K(X)\right.$ and $\left.A K=K A\right\}$, there is a $K \in K(X)$ such that $A K=K A$ and $\lambda \notin \sigma_{g}(A+K)$. Thus, $R(A+K-\lambda)$ is closed and $k(A+K-\lambda)=0$. Adding the operator $-K$ to $A+K-\lambda$, we see that $R(A-\lambda)$ is closed and $k(A-\lambda)<\infty([5$, Theorem 5.9]). Hence $A-\lambda \in V(X)$. To prove the converse suppose that $A-\lambda \in V(X)$. If $k(A-\lambda)=0$, then $\lambda \notin \sigma_{g}(A)$ and the proof is complete. If $0<k(A-\lambda)$, then by Theorem 2.1 we conclude that the space $X$ decomposes into a direct sum of two closed subspaces $X_{0}$ and $X_{1}$. These subspaces are $(A-\lambda)$-invariant, hence $A$-invariant, and have the following properties: The space $X_{1}$ is finite dimensional (and $A-\lambda$ is nilpotent on it). If $A_{0}$ is the restriction of $A$ to $X_{0}$ considered as an operator from $X_{0}$ into itself then $k\left(A_{0}-\lambda\right)=0$. Let $F$ be the finite rank operator defined by $F=I$ on $X_{1}, F=0$ on $X_{0}$. Hence, $A F=F A$ and $R(A+F-\lambda)$ is closed. Since $A-\lambda$ is nilpotent on $X_{1}$ we have $N(A+F-\lambda)=N\left(A_{0}-\lambda\right) \subset R\left(\left(A_{0}-\lambda\right)^{\infty}\right) \subset R\left(\left(A_{0}-\lambda\right)^{\infty}\right) \oplus X_{1}=R\left((A+F-\lambda)^{\infty}\right.$. Thus, $k(A+F-\lambda)=0$, and $\lambda \notin \sigma_{g}(A+K)$. This completes the proof.

Corollary 3.2 $\bigcap\left\{\sigma_{\theta}(A+K): K \in F(X)\right.$ and $\left.A K=K A\right\}=\sigma_{\theta b}(A)$.

Proof. Inclusion ' $\supset$ ' is obvious. Suppose that $\lambda \notin \sigma_{g b}(A)$. From the proof of Theorem 3.1, there exists a finite rank operator $F$ in $B(X)$ such that $A F=F A$ and $\lambda \notin \sigma_{g}(A+F)$, which proves the inclusion ' $\subset$ '. This completes the proof.

Let us point out that Theorem 3.1 and its corollary can be proved without using Theorem 2.1, but instead by using Kaashoek's [6, Theorem 3.2]. 
Corollary 3.3. $\lambda \in \sigma_{g}(A) \backslash \sigma_{g b}(A)$ if and only if $\lambda$ is an isolated point of $\sigma_{\theta}(A)$, $0<k(A-\lambda)<\infty$ and $R(A-\lambda)$ is closed.

Proof. This follows from Theorem 3.1, [5, Theorem 4.7] and [6, Theorem 4.1].

The polynomial hull $\hat{E}$ of a compact subset $E$ of the complex plane $\mathbb{C}$ is the complement of the unbounded component of $\mathbb{C} \backslash E$. Given a compact subset $E$ of the plane, a hole of $E$ is a component of $\hat{E} \backslash E$. If $F$ is another compact set such that $\partial E \subset F \subset E$, it follows that $\partial E \subset \partial F, \hat{E}=\hat{F}$ and $E$ can be obtained from $F$ by filling in some holes of $F$. (Here and in what follows $\partial E$ denotes the boundary of the set $E$.)

Corollary 3.4. Let $A \in B(X)$. Then

(i) $\sigma_{g b}(A) \subset \sigma_{e k}(A)$,

(ii) $\partial \sigma_{e k}(A) \subset \partial \sigma_{g b}(A)$ and $\sigma_{g b}(A)$ is nonempty,

(iii) $\hat{\sigma}_{g b}=\hat{\sigma}_{e k}(A)$,

(iv) $\sigma_{e k}(A)$ can be obtained from $\sigma_{g b}(A)$ by filling in some holes of $\sigma_{g b}(A)$,

(v) if $\sigma_{g b}(A)$ is connected, $\sigma_{e k}(A)$ is connected.

Proof. It is sufficient to prove (ii). It is well known that $\sigma_{e k}(A)$ is nonempty and compact. Suppose $\lambda_{0} \in \partial \sigma_{e k}(A)$ and $\lambda_{0} \notin \sigma_{g b}(A)$. Hence, $k\left(A-\lambda_{0}\right)<\infty$ and $R\left(A-\lambda_{0}\right)$ is closed. Now, we know that there exists an $\varepsilon>0$ such that $0<\left|\lambda_{0}-\lambda\right|<\varepsilon$ implies that $R(A-\lambda)$ is closed and $\alpha(A-\lambda)$ and $\beta(A-\lambda)$ are constant, i.e., $\alpha(A-\lambda)=\alpha\left(A-\lambda_{0}\right)-$ $k\left(A-\lambda_{0}\right)$ and $\beta(A-\lambda)=\beta\left(A-\lambda_{0}\right)-k\left(A-\lambda_{0}\right)\left(\left[6\right.\right.$, Theorem 4.1]). Thus $A-\lambda_{0} \in \Phi_{+}(X) \cup$ $\Phi_{-}(X)$, which is a contradiction. This completes the proof.

Corollary 3.5. Let $A^{*}$ be the adjoint operator of $A \in B(X)$. Then $\sigma_{g b}(A)=\sigma_{g b}\left(A^{*}\right)$.

Proof. This follows from Theorem 3.1, [15, Theorem 2] and [5, Theorem 3.7].

Recall that $a(A)$, the ascent of $A$, is the smallest non-negative integer $n$ such that $N\left(A^{n}\right)=N\left(A^{n+1}\right)$. If no such $n$ exists, then $a(A)=\infty$. Let $A_{\mid M}$ denotes the restriction of $A$ to the subspace $M$ of $X$.

Corollary 3.6. Let $A \in V(X)$. Then the following statements are equivalent:

(i) $A=V+F$, where $\alpha(V)=0, F$ is finite rank and $V F=F V$;

(ii) there exists a finite rank projection $P$ commuting with $A$ such that $\alpha\left(A_{\mid N(P)}\right)=0$;

(iii) there exists $\varepsilon>0$ such that $\alpha(A+\lambda)=0$ for $0<|\lambda|<\varepsilon$;

(iv) $a(V)<\infty$.

Proof. If $A$ satisfies any condition among (i)-(iv), then $A \in \Phi_{+}(X)$ and $i(A) \leqq 0([11$, Lemma 2.5], [6, Theorem 4.1]). Thus, the proof follows by [17, Corollary 2.7] or [19, Proposition 2.6]. 
Let $\mathscr{P}(X)$ denote the set of all bounded projections $P$ in $X$ such that $\operatorname{codim} P(X)$ is finite. The compression $A_{P}$ is a bounded linear operator on the closed subspace $P X$ defined by $A_{P} y=P A y$ for each $y$ in $P X$. Consequently, $\sigma_{g}\left(A_{P}\right)$ is the generalized spectrum of this operator on the Banach space $P X$.

Theorem 3.7. For every bounded linear operator on a Banach space $X$ we have

$$
\sigma_{g b}(A)=\bigcap\left\{\sigma_{g}\left(A_{P}\right): P \in \mathscr{P}(X) \text { and } P A=A P\right\} .
$$

Proof. Suppose that $\lambda$ is not in $\sigma_{g b}(A)$. Then $R(A-\lambda)$ is closed and $k(A-\lambda)<\infty$, i.e., $A-\lambda \in V(X)$. Consequently, by Theorem 2.1 the space $X$ is the direct sum of two closed subspaces $X_{0}$ and $X_{1}$ which are $A$-invariant and have the following properties: The space $X_{1}$ is finite dimensional (possibly zero) and $A-\lambda$ is nilpotent on it. If $A_{0}$ denotes the restriction of $A$ to $X_{0}$ considered as an operator from $X_{0}$ into itself (and $P$ the projection of $X$ onto $X_{0}$ along $\left.X_{1}\right)$, then $N\left(\left(A_{0}-\lambda\right)_{P}\right) \subset R\left(\left(A_{0}-\lambda\right)_{P}^{\infty}\right)$. Let us remark that $P A=A P, P \in \mathscr{P}(X)$ and $R\left((A-\lambda)_{P}\right)$ is closed (Remark 2.2). Thus $\lambda \notin \sigma_{g}\left(A_{P}\right)$. This proves that $\sigma_{g b}(A) \supset \bigcap\left\{\sigma_{g}\left(A_{P}\right): P \in \mathscr{P}(X)\right.$ and $\left.A P=P A\right\}$.

To prove the converse inclusion, suppose that $\lambda$ is not in $\sigma_{g}\left(A_{P}\right)$ for some $P \in \mathscr{P}(X)$ such that $A P=P A$. Thus $R((A-\lambda) P)$ is closed and $k((A-\lambda) P)=0$. Since $A-\lambda=$ $(A-\lambda) P+(A-\lambda)(I-P)$ and $(A-\lambda)(I-P)$ is a finite rank operator, we conclude that $\lambda \notin \sigma_{g b}(A)([5$, Theorem 5.9]). The proof is complete.

Let us remark that it has been observed by Zemánek that for Browder's essential approximate point spectrum of $A$ we have $\sigma_{a b}(A)=\bigcap\left\{\sigma_{a}\left(A_{P}\right): P \in \mathscr{P}(X)\right.$ and $\left.A P=P A\right\}$ ([21, Theorem 3]).

\section{Spectral mapping theorem for $\sigma_{g b}(A)$}

Theorem 4.1. If $A$ is any operator and $p$ is any polynomial, then

$$
\sigma_{g b}(p(A))=p\left(\sigma_{g b}(A)\right)
$$

Proof. Let $\lambda \notin p\left(\sigma_{g b}(A)\right)$ and $p(t)-\lambda=c\left(t-\lambda_{1}\right)^{m_{1}} \ldots\left(t-\lambda_{k}\right)^{m_{k}}$ with $m_{i}$ integers, $c \neq 0$ and $\lambda_{i} \neq \lambda_{j}$ for $i \neq j$. Thus, $p(A)-\lambda=c\left(A-\lambda_{1}\right)^{m_{1}} \ldots\left(A-\lambda_{k}\right)^{m_{k}}$ and $\lambda_{i} \notin \sigma_{g b}(A)$ for $i=1, \ldots, k$. Consequently, we have that $R\left(A-\lambda_{i}\right)$ is closed and $k\left(A-\lambda_{i}\right)<\infty$, for $i=1, \ldots, k$. From $\left[5\right.$, Theorem 3.8], we know that $R\left(\left(A-\lambda_{i}\right)^{m_{i}}\right)$ is closed and by [5, Lemma 3.11$] k\left(\left(A-\lambda_{i}\right)^{m_{i}}\right)<\infty$ for $i=1, \ldots, k$. Let us remark that by $([4$, Corollary]) we have that

$$
R(p(A)-\lambda)=R\left(\left(A-\lambda_{1}\right)^{m_{1}}\right) \cap \ldots \cap R\left(\left(A-\lambda_{k}\right)^{m_{k}}\right)
$$

and

$$
N(p(A)-\lambda)=N\left(\left(A-\lambda_{1}\right)^{m_{1}}\right) \oplus \ldots \oplus N\left(\left(A-\lambda_{k}\right)^{m_{k}}\right)
$$


Thus $R(p(A)-\lambda)$ is closed. Further, by ([5, Lemma 2.1(a)]) and the elementary fact that if $\lambda \neq 0$, then $N\left((A+\lambda)^{\infty}\right) \subset R\left(A^{\infty}\right)$, for each integer $n$ we have

$$
\begin{aligned}
& \frac{N(p(A)-\lambda)}{N(p(A)-\lambda) \cap R\left((p(A)-\lambda)^{n}\right)} \\
& =\frac{N\left(\left(A-\lambda_{1}\right)^{m_{1}}\right) \oplus \ldots \oplus N\left(\left(A-\lambda_{k}\right)^{m_{k}}\right)}{\left(N\left(\left(A-\lambda_{1}\right)^{m_{1}}\right) \oplus \ldots \oplus N\left(\left(A-\lambda_{k}\right)^{m_{k}}\right)\right) \cap R\left(\left(A-\lambda_{1}\right)^{m_{1} n}\right) \cap \ldots \cap R\left(\left(A-\lambda_{k}\right)^{m_{k} n}\right)} \\
& =\frac{N\left(\left(A-\lambda_{1}\right)^{m_{1}}\right) \oplus \ldots \oplus N\left(\left(A-\lambda_{k}\right)^{m_{k}}\right)}{N\left(\left(A-\lambda_{1}\right)^{m_{1}}\right) \cap R\left(\left(A-\lambda_{1}\right)^{m_{1 n}}\right) \oplus \ldots \oplus N\left(\left(A-\lambda_{k}\right)^{m_{k} n}\right) \cap R\left(\left(A-\lambda_{k}\right)^{m_{k} n}\right)^{.}}
\end{aligned}
$$

Thus,

$$
\operatorname{dim} \frac{N(p(A)-\lambda)}{N(p(A)-\lambda) \cap R\left((p(A)-\lambda)^{n}\right)} \leqq \sum_{i=1}^{n} \operatorname{dim} \frac{N\left(\left(A-\lambda_{i}\right)^{m_{i}}\right)}{N\left(\left(A-\lambda_{i}\right)^{m_{i}}\right) \cap R\left(\left(A-\lambda_{i}\right)^{m_{i n}}\right)}
$$

and by [5, Theorem 3.7] it follows that $k(p(A)-\lambda) \leqq \Sigma k\left(\left(A-\lambda_{i}\right)^{m_{i}}\right)$. Hence, $\lambda \notin \sigma_{g b}(p(A))$.

We now turn to the proof of the opposite inclusion. Suppose that $\lambda \in p\left(\sigma_{g b}(A)\right)$ and $\lambda \notin \sigma_{g b}(p(A))$. By the definition of $\sigma_{g b}(A)$, we have that $R(p(A)-\lambda)$ is closed and $k(p(A)-\lambda)<\infty$. By $([4$, Corollary (iii) $])$ we know that $R\left(\left(A-\lambda_{i}\right)^{m_{i}}\right)$ is closed for $i=1, \ldots, k$. Since $N\left(\left(A-\lambda_{i}\right)^{m_{i}}\right) \subset N(p(A)-\lambda)$, and for each positive integer $m$ and $n, N\left(\left(A-\lambda_{i}\right)^{m}\right) \subset R\left(\left(A-\lambda_{j}\right)^{n}\right),(i \neq j)$, then by [7, Lemma 2.3] we have

$$
\operatorname{dim} \frac{N\left(A-\lambda_{i}\right)}{N\left(A-\lambda_{i}\right) \cap R\left(\left(A-\lambda_{i}\right)^{m_{i} n}\right)} \leqq \operatorname{dim} \frac{N(p(A)-\lambda)}{N(p(A)-\lambda) \cap R\left((p(A)-\lambda)^{n}\right)} .
$$

This shows that $k\left(A-\lambda_{i}\right)<\infty\left(\left[5\right.\right.$, Theorem 3.7]), and by [5, Theorem 3.8] $R\left(A-\lambda_{i}\right)$ is closed. According to this, we have that $\lambda_{i} \notin \sigma_{g b}(A),(i=1, \ldots, k)$, which provides a contradiction. The proof is complete.

Theorem 4.2. Let $A \in B(X)$, and let $D$ be an open neighbourhood of $\sigma(A)$. If $f$ is $a$ rational function on $D$ with no poles in $D$, then

$$
\sigma_{g b}(f(A))=f\left(\sigma_{g b}(A)\right)
$$

Proof. We can write $f=p / q$, where $p$ and $q$ are polynomials and $q$ has no zeros in $D$. Hence, $0 \notin q(\sigma(A)), q(A)$ is invertible and $f(A)=p(A) q(A)^{-1}=q(A)^{-1} p(A)$. For each $\lambda \in \mathbb{C}$ we now write, assuming that $p / q$ is not constant,

$$
\frac{p}{q}-\lambda=\frac{p-\lambda q}{q}=\frac{1}{q} c\left(z-\lambda_{1}\right)\left(z-\lambda_{2}\right) \ldots\left(z-\lambda_{n}\right) .
$$


Hence

$$
f(A)-\lambda=q(A)^{-1} c\left(A-\lambda_{1}\right)\left(A-\lambda_{2}\right) \ldots\left(A-\lambda_{n}\right)
$$

and the proof of Theorem 4.2 follows by Theorem 4.1 .

Let $\left(G_{n}\right)$ be a sequence of compact subsets of $\mathbb{C}$. The limit superior, $\lim \sup G_{n}$, is the set of all $\lambda$ in $\mathbb{C}$ such that every neighbourhood of $\lambda$ intersects infinitely many $G_{n}$. To show that if $f$ is an analytic function defined on a neighbourhood of $\sigma(A)$, then $f\left(\sigma_{g b}(A)\right)=\sigma_{g b}(f(A))$ we shall prove the following statement.

Theorem 4.3. Let $A, A_{n} \in B(X), A_{n} \rightarrow A$ and $A A_{n}=A_{n} A$ for each positive integer $n$. Then

(i) $\lim \sup \sigma_{g}\left(A_{n}\right) \subset \sigma_{g}(A)$,

(ii) $\lim \sup \sigma_{g b}\left(A_{n}\right) \subset \sigma_{g b}(A)$.

Proof. (i) It is enough to show that if $0 \notin \sigma_{g}(A)$, then $0 \notin \lim \sup \sigma_{g}\left(A_{n}\right)$. Suppose that $0 \notin \sigma_{g}(A)$. Then $R(A)$ is closed and $k(A)=0$. Then, by [5, Lemma 4.2] we know that there exists an $\varepsilon>0$ and an integer $n_{0}$ such that $R\left(A_{n}-\lambda\right)$ are closed for $n \geqq n_{0}$ and $k\left(A_{n}-\lambda\right)=0$ for $|\lambda|<\varepsilon$. Therefore, for $n \geqq n_{0}$ we see that $\sigma_{\theta}\left(A_{n}\right) \cap\{\lambda \in \mathbb{C}:|\lambda|<\varepsilon\}$ is empty. Thus, we have that $0 \notin \lim \sup \sigma_{g}\left(A_{n}\right)$.

(ii) To prove (ii), it is enough to show that if $0 \notin \sigma_{g b}(A)$, then $0 \notin \limsup \sigma_{g b}\left(A_{n}\right)$. If $0 \notin \sigma_{g}(A)$, then by (i) we know that $0 \notin \lim \sup \sigma_{g}\left(A_{n}\right)$, and $0 \notin \lim \sup \sigma_{g b}\left(A_{n}\right)$. If $0 \in \sigma_{g}(A) \backslash$ $\sigma_{g b}(A)$ then $R(A)$ is closed and $0<k(A)<\infty$. Consequently, by [5, Theorem 4.10(a)] there exists an $\varepsilon>0$ and an integer $n_{0}$ such that $R\left(A_{n}-\lambda\right)$ are closed and $k\left(A_{n}-\lambda\right)<\infty$ for $|\lambda|<\varepsilon$ and $n \geqq n_{0}$. Therefore, for $n \geqq n_{0}$ we see that $\sigma_{g b}\left(A_{n}\right) \cap\{\lambda \in \mathbb{C}:|\lambda|<\varepsilon\}$ is empty. Thus we have $0 \notin \lim \sup \sigma_{g b}\left(A_{n}\right)$, and the proof is complete.

Remark 4.4. Let us remark that the commutativity conditions in Theorem 4.3 are necessary. Examples in which $\sigma_{g}$ and $\sigma_{g b}$ are not upper semi-continuous can be constructed using the result of Goldman [3, Theorem 1]. In fact, if $A \in V(X)\left(V_{0}(X)\right)$, $\alpha(A)=\infty$ and $\beta(A)=\infty$, by [3, Theorem 1] there exists a sequence $A_{n}$ of linear bounded operators on $X$, with non-closed ranges, such that $A_{n} \rightarrow A$. Thus, we have that $0 \notin \sigma_{g b}(A)$ $\left(\sigma_{g}(A)\right)$ and $0 \in \sigma_{g b}\left(A_{n}\right)$ for each $n$ (which implies $0 \in \lim \sup \sigma_{g b}\left(A_{n}\right)$ ).

Theorem 4.5. Let $A \in B(X)$ and let $f$ be an analytic function defined on a neighbourhood of $\sigma(A)$. Then

$$
f\left(\sigma_{g b}(A)\right)=\sigma_{g b}(f(A))
$$

Proof. Let $D$ be a neighbourhood of $\sigma(A)$, and let $\left(f_{n}(t)\right)$ be a sequence of rational functions, with no poles in $D$, converging to $f(t)$ on $D$. We have

$$
f\left(\sigma_{g b}(A)\right)=\lim f_{n}\left(\sigma_{g b}(A)\right)
$$




$$
\begin{array}{ll}
=\lim \sup \sigma_{g b}\left(f_{n}(A)\right) & \text { (by Theorem 4.2) } \\
\subset \sigma_{g b}(f(A)) & \text { (by Theorem 4.3(ii)). }
\end{array}
$$

To prove the converse suppose that $\mu \notin f\left(\sigma_{g b}(A)\right)$. Thus, for each $\lambda \in \sigma_{g b}(A)$ we have that $f(\lambda)-\mu \neq 0$. Set $g(\lambda)=f(\lambda)-\mu$. If $g(\lambda) \neq 0$ for each $\lambda \in \sigma(A)$, then $g(A)$ is invertible, and $\mu \notin \sigma(f(A))$. Thus $\mu \notin \sigma_{g b}(f(A))$. Now suppose that $g(\lambda)$ has zeros of order $n_{i}$ at $\lambda_{i} \in \sigma(A), i=1, \ldots, k$. Then

$$
g(\lambda)=\prod_{i=1}^{k}\left(\lambda-\lambda_{i}\right)^{n_{i}} h(\lambda) \text { and } h(\lambda) \neq 0 \text { for each } \lambda \in \sigma(A)
$$

Set

$$
p(\lambda)=\prod_{i=1}^{k}\left(\lambda-\lambda_{i}\right)^{n_{i}}
$$

By Theorem 4.1, we know that $0 \notin \sigma_{g b}(p(A))$. Then $R(p(A))$ is closed and $k(p(A))<\infty$. Consequently, since $h(A)$ is an invertible operator commuting with $p(A)$, it is easy to see that $g(A)=p(A) h(A)$ has closed range and $k(g(A))<\infty$. Thus, we have that $\mu \notin \sigma_{g b}(f(A))$, i.e., $\sigma_{g b}(f(A)) \subset f\left(\sigma_{g b}(A)\right)$. This completes the proof of the theorem.

\section{Connected components of $\mathbb{C} \backslash \sigma_{g b}(A)$}

If $A \in B(X)$, then $\mathbb{C} \backslash \sigma_{g b}(A)$ is an open set in the complex plane $\mathbb{C}$. Let $U$ be a connected component of $\mathbb{C} \backslash \sigma_{g b}(A)$ and $G=\left\{\lambda \in \mathbb{C} \backslash \sigma_{g b}(A): k(A-\lambda) \neq 0\right\}$. By [6, Theorem 4.1] we know that $G$ has no accumulation point in $\mathbb{C} \backslash \sigma_{g b}(A)$. A complex number $\lambda \in G \cap U$ is called a jumping point in $U$.

Remark 5.1. If $\lambda$ is a jumping point in $U$, then by Theorem 2.1 (ii), there is an $A$-invariant finite dimensional subspace $N_{\lambda}$ in $X$ such that $A-\lambda$ is nilpotent on it. Consistent with the matrix case we define the (algebraic) multiplicity of the jumping point $\lambda$ to be $\operatorname{dim} N_{\lambda}$. If $U$ is a connected component of the semi-Fredholm region of $A$, then our definition of the multiplicity of the jumping point $\lambda$ in $U$ is consistent with the definition in [18, p. 232] and [22, p. 449].

Theorem 5.2. Let $A \in B(X)$ and let $U$ and $G$ be as above. Then the functions

$$
\lambda \rightarrow N\left((A-\lambda)^{\infty}\right)+R\left((A-\lambda)^{\infty}\right) \text { and } \lambda \rightarrow N\left((A-\lambda)^{\infty}\right) \cap R\left((A-\lambda)^{\infty}\right)
$$

are constant on $U$, while the functions

$$
\lambda \rightarrow R\left((A-\lambda)^{\infty}\right) \text { and } \lambda \rightarrow N\left((A-\lambda)^{\infty}\right)
$$


are constant on $U \backslash G$.

Proof. The proof follows from [9, Theorem 3] and ([5, Theorem 4.7(d), (e); Lemma 4.2(d), (e); Lemma 3.6]).

Remark 5.3. Let us remark that by [5, Lemma 3.6(a)] and Theorem 5.2 we have that

$$
\begin{aligned}
\left.R\left((A-\lambda)^{\infty}\right)+N(A-\lambda)^{\infty}\right) & =R\left((A-\lambda)^{\infty}\right)+\operatorname{cl}\left(N\left((A-\lambda)^{\infty}\right)\right) \\
& =R\left((A-\lambda)^{\infty}\right) \oplus N_{\lambda}=W
\end{aligned}
$$

for each $\lambda \in U$, where $N_{\lambda}$ is a finite dimensional subspace, $N_{\lambda}$ is $A$-invariant and $(A-\lambda)_{\mid N_{\lambda}}$ is nilpotent on it. Thus, $W$ is closed, hence a Banach subspace in $X([5$, Theorem 3.8]). The restriction of $A$ to the subspace $W$ has been studied in [16] and [19].

Theorem 5.4. If $A \in V(X)$, set $v_{0}(A)=\sup \left\{\varepsilon>0: A-\lambda \in V_{0}(X)\right.$ for $\left.0<|\lambda|<\varepsilon\right\}$ and $v(A)=\sup \{\varepsilon>0: A-\lambda \in V(X)$ for $|\lambda|<\varepsilon\}$. Then

$$
v(A)=\sup \left\{v_{0}(A+F): F \in F(X) \text { and } A F=F A\right\} \text {. }
$$

Proof. Let $F \in F(X)$ and $A F=F A$. Then $A+F \in V(X)([5$, Theorem 5.9]). If $|\lambda|<$ $v_{0}(A+F)$, again by $([5$, Theorem 5.9]) we have that $A-\lambda=(A+F-\lambda)-F \in V(X)$. Hence, $v_{0}(A+F) \leqq v(A)$, i.e., $\sup \left\{v_{0}(A+F): F \in F(X)\right.$ and $\left.A F=F A\right\} \leqq v(A)$.

To prove the other inequality suppose that $\varepsilon>0$, and let $p$ denote the total multiplicity of the jumps having absolute value less than $v(A)-\varepsilon$. As in the proof of $[18$, Theorem 1.1(II)] (using Theorem 2.1 instead of Kato's decomposition theorem [9, Theorem 4]) we conclude that the space $X$ decomposes into the direct sum of two closed subspaces $Z$ and $Y$ which are $A$-invariant, $\operatorname{dim} Z=p$ and $Z$ is the direct sum of the finite dimensional summands at the jumping points $\lambda_{1}(A), \ldots, \lambda_{p}(A)$ (where each jump appears consecutively according to its multiplicity). Let $P^{2}=P \in B(X)$ be the idempotent with $R(P)=Z$ and $N(P)=Y$. It is clear that $P \in F(X)$ and $A P=P A$. Set $F=\alpha P$, with $|\alpha|>\|A\|+v(T)$. Now, as in the proof of [20, Theorem 7.1], for each $\lambda$ with $|\lambda|<v(A)-\varepsilon$ we have that $R(A+F-\lambda)$ is closed and $N(A+F-\lambda) \subset R\left((A+F-\lambda)^{\infty}\right)$. Thus, $v_{0}(A+F) \geqq v(A)-\varepsilon$, and the proof is complete.

Lemma 5.5. Let $A \in B(X)$ and let $U, G$ and $W$ be as above. Then:

(i) $(A-\lambda)_{\mid W} \in \Phi_{-}(W)$ for each $\lambda \in U$;

(ii) if $\lambda \in U$, then $\lambda \in U \cap G$ if and only if $\lambda$ is a jumping point in the semi-Fredholm region of $A_{\mid W}$.

Proof. Let $\lambda \in U$. Then $W=R\left((A-\lambda)^{\infty}\right) \oplus N_{\lambda}$ (Remark 5.3). By [5, Theorem 3.4] we 
have that $(A-\lambda) W=(A-\lambda) R\left((A-\lambda)^{\infty}\right) \oplus(A-\lambda) N_{\lambda}=R\left((A-\lambda)^{\infty}\right) \oplus(A-\lambda) N_{\lambda}$. Thus, $(A-\lambda)_{\mid W} \in \Phi_{-}(W)$, which proves (i). (ii) follows by Remark 5.1 and (i).

For a technical reason we suppose that the connected component $U$ contains zero. Then the points in $G \cap U$ can be ordered in such a way that

$$
\left|\lambda_{1}(A)\right| \leqq\left|\lambda_{2}(A)\right| \leqq \ldots<v(A)
$$

where each jump appears consecutively according to its multiplicity. If there are only $p$ $(=0,1,2, \ldots)$ such jumps, we put $\left|\lambda_{p+1}(A)\right|=\left|\lambda_{p+2}(A)\right|=v(A)$.

Let $S$ denote the closed unit ball of $X$. Let

$$
q(A)=\sup \{\varepsilon \geqq 0: A S \supset \varepsilon S\}
$$

be the surjection modulus of $A$. For each $r=1,2, \ldots$ we define

$$
q_{r}(A)=\sup \{q(A+F): \text { rank } F<r\} .
$$

Theorem 5.6. Let $A \in V(X), 0 \in U$, and let $U, G$ and $W$ be as above. Then for each jumping point $\lambda_{r}(A), r=1,2, \ldots$ we have

$$
\left|\lambda_{r}(A)\right|=\lim _{k} q_{r}\left(\left(A_{\mid W}\right)^{k}\right)^{1 / k}
$$

Proof. By Lemma 5.5 we know that $(A-\lambda)_{\mid W} \in \Phi_{-}(W)$ for each $\lambda \in U$, and that $\lambda_{r}(A)$, $r=1,2, \ldots$ are jumps (with the same multiplicity) in the semi-Fredholm region of $A_{\mid W}$ (Remark 5.1). Thus, the proof of the theorem follows by [18, Theorem 1.1, pp. 232-233] (since the stability index of the semi-Fredholm operator $A_{\mid W}$ is 0 ).

If $T$ is a linear operator from a Banach space $X$ to another Banach space $Y$, then the reduced minimum modulus of $T$ is defined by

$$
\gamma(T)=\inf \{\|T x\|: \operatorname{dist}(x, N(T))=1\}
$$

For each $r=1,2, \ldots$ we put

$$
\gamma_{r}^{-}(T)=\sup \left\{\gamma\left(Q_{V} T\right): \operatorname{dim} V<r\right\},
$$

where $Q_{V}$ is the canonical map of $X$ onto the quotient space $X / V$. Now, we have:

Corollary 5.7. Let $A \in B(X)$ and let $\lambda_{r}(A), r=1,2, \ldots, U$ and $W$ be as above. Then for each jumping point $\lambda_{r}(A), r=1,2, \ldots$ we have 


$$
\left|\lambda_{\rho+r}(A)\right|=\lim _{k} \gamma_{r}^{-}\left(\left(A_{\mid W}\right)^{k}\right)^{1 / k}
$$

where $\rho$ is the multiplicity of the jump at zero.

Proof. The proof follows by [22, Theorem 1, p. 451], Lemma 5.5 and Theorem 5.6.

Corollary 5.8. If $A \in V(X)$, then

$$
v_{0}(A)=\lim _{k} \gamma\left(\left(A_{\mid W}\right)^{k}\right)^{1 / k}
$$

Proof. This follows from Corollary 5.7.

Let $A \in B(X)$ be a semi-Fredholm operator. Then the semi-Fredholm radius $s(A)$ of $A$ is the supremum of all $\varepsilon \geqq 0$ such that the operator $A-\lambda$ is semi-Fredholm for $|\lambda|<\varepsilon$.

Corollary 5.9. Let $A \in V(X)$ and let $\lambda_{r}(A), r=1,2, \ldots, U$ and $W$ be as above. Then:

(i) if there is a finite number of jumps, then $v(A) \leqq s\left(A_{\mid W}\right)$.

(ii) if there is an infinite number of jumps, then $v(A)=s\left(A_{\mid W}\right)$.

Proof. This follows by Lemma 5.5 and [6, Theorem 4.1].

We would like to finish this paper with the following questions:

Question 1. If $A \in V(X)$, must $\lim _{k} \gamma\left(A^{k}\right)^{1 / k}=v_{0}(0)$ ?

(Let us remark that the limit exists (by Theorem 2.1 and the proof of [2, Theorem 2]). If $X$ is a Hilbert space, then the answer to the Question 1 is positive (see [1, Theorem 3.2, Corollary 3.4] or [13, Théorème 3.1, Corollaire 3.9]).)

Question 2. If $A, B \in B(X)$ and $A B=B A \in V(X)$, must $A, B \in V(X)$ ?

(Let us remark that if $A, B \in B(X)$ and $A B=B A \in V_{0}(X)$, then $A, B \in V_{0}(X)([13$, Lemma 4.15]).)

Question 3. If $A, B \in B(X), A B=B A$ and $B$ is a quasinilpotent operator, must

$$
\sigma_{\theta b}(A+B)=\sigma_{g b}(A) ?
$$

(Recall that if $X$ is a Hilbert space, $A, B \in B(X), A B=B A$ and $B$ is a quasinilpotent operator, then $\sigma_{g}(A+B)=\sigma_{g}(A)([13$, Théorème 4.8].)

Question 4. If $A, B \in V(X)$ (or $V_{0}(X)$ ) and $A B=B A$, must $A B \in V(X)$ (or $V_{0}(X)$ ), and possibly $k(A B) \leqq k(A)+k(B)$ ? 
Acknowledgements. I am grateful to Professor Laura Burlando for the examples in the Remark 4.4, and to Professor Jean-Philippe Labrousse for comments and verification of the proof of Theorem 2.1. The author also thanks the referee for helpful comments and suggestions concerning the paper.

\section{REFERENCES}

1. C. Apostol, The reduced minimum modulus, Michigan Math. J. 32 (1985), 279-294.

2. K.-H. Forster and M. A. KaAshoeK, The asymptotic behaviour of the reduced minimum modulus of a Fredholm operator, Proc. Amer. Math. Soc. 49 (1975), 123-131.

3. M. A. Goldman, On the stability of normal solvability of linear equations (Russian), Dokl. Akad. Nauk SSSR. 100 (1955), 201-204.

4. M. González, Null spaces and ranges of polynomials of operators, Publ. Mat. 32 (1988), 167-170.

5. S. Grabiner, Uniform ascent and descent of bounded operators, J. Math. Soc. Japan 34 (1982), 317-337.

6. M. A. KaASHOEK, Stability theorems for closed linear operators, Indag. Math. 27 (1965), 452-466.

7. M. A. KaAshoek, Ascent, descent, nullity and defect, a note on a paper by A. E. Taylor, Math. Ann. 172 (1967), 105-115.

8. S. N. KRAChKovskil, On the extended region of the singularity of the operator $T_{2}=E-\lambda A$ (Russian), Dokl. Akad. Nauk SSSR 96 (1954), 1101-1104.

9. T. Kato, Perturbation theory for nullity, deficiency and other quantities of linear operators, J. Analyse Math. 6 (1958), 261-322.

10. J. P. Labrousse, Les opérateurs quasi-Fredholm: Une généralisation des opérateurs semi-Fredholm, Rend. Circ. Mat. Palermo (2) 29 (1980), 161-258.

11. D. C. LAY, Spectral analysis using ascent, descent, nullity and defect, Math. Ann. 184 (1970), 197-214.

12. A. S. Markus, On one theorem of F. Reisz (Russian), Uch. Zap. Kishinev. Gos. Univ. 17 (1955), 73-76.

13. M. Мвекhta, Résolvant généralisé et théorie spectrale, J. Operator Theory 21 (1989), 69-105.

14. S. M. Nixolskn, Linear equations in normed linear spaces (Russian), Bull. Acad. Sci. URSS Ser. Math. 7 (1943), 147-166.

15. R. K. Oliver, Note on a duality relation of Kaashoek, Indag. Math. 28 (1966), 364-368.

16. M. Ó SEarcóid and T. T. WEst, Continuity of the generalized kernel and range of semi-Fredholm operators, Math. Proc. Cambridge Philos. Soc. 105 (1989), 513-522.

17. V. RaKoĆvić, Approximate point spectrum and commuting compact perturbations, Glasgow Math. J. 28 (1986), 193-198.

18. V. RakóceviC and J. Zemánek, Lower s-numbers and their asymptotic behaviour, Studia Math. 91 (1988), 231-239.

19. T. T. West, A Riesz-Schauder theorem for semi-Fredholm operators, Proc. Roy. Irish Acad. Sect. A 87 (1987), 137-146. 
20. J. ZemÁneK, Geometric characteristics of semi-Friedholm operators and their asymptotic behaviour, Studia Math. 80 (1984), 219-234.

21. J. Zemánek, Compressions and the Weyl-Browder spectra, Proc. Roy. Irish Acad. Sect. A 86 (1986), 57-62.

22. J. ZemÁneK, The reduced minimum modulus and the spectrum, Integral Equations Operator Theory 12 (1989), 449-454.

University of Niš, Faculty of Philosophy

Department of Mathematics

Ćirila and MetodiJa 2

18000 Nis

Yugoslavia 